\title{
Then there were none!
}

\author{
Veronica J. James $^{1 *}$, Judith M. O’Malley Ford ${ }^{2}$ and Jason Buttigieg ${ }^{3}$ \\ ${ }^{1}$ Research School of Chemistry, Australian National University, Australia \\ ${ }^{2}$ Kedron 7Day Medical Centre, Australia \\ ${ }^{3}$ Faculty of Medicine, Nursing and Health Sciences, Monash University, Australia
}

\begin{abstract}
Fibre diffraction introduces an exciting new approach to cancer diagnosis. Not only is this technique able to detect the cancers accurately[sensitivity $100 \%$ - no false negatives] but also the specific pattern changes are present from their very beginning of the cancers. In fact fibre diffraction can detect the presence of cancers much earlier than any other test. This enables treatments when cancers are extremely small and low grade. However since the changes noted are caused by the cancers themselves, if the cancers are successfully removed, so too will changes in the diffraction pattern disappear, thus confirming the cure.
\end{abstract}

\section{Introduction}

In our very first breast cancer diagnostic study of hair [1], one patient who, the surgeon believed had been cured by a mastectomy some seven years earlier, showed no "ring of change." In subsequent years, eight women, who were believed to have been cured, were tested yearly and only one of these showed the breast cancer ring of change in the first year. A subsequent check revealed that pathology had discovered that the breast cancer type of her mastectomy specimen was different from that obtained in her original breast biopsy specimen. A follow up examination revealed the presence of the second tumour. When this was removed, her diffraction tests no longer showed the "breast cancer" ring of change.

Following a lumpectomy another patient was persuaded to have chemotherapy and sent me all her hair when it fell out following the second round of chemotherapy, 88 days after her lumpectomy. Using her hair and basing my calculations on a growth of $3.5 \mathrm{~mm}$ per week [2], pattern (i) in Figure 1, taken $4.5 \mathrm{~cm}$ from the follicle becomes the pattern for 2 days before the lumpectomy. This diffraction pattern 1(i) clearly shows the breast cancer ring, indicated by the arrow. Pattern 1(ii), where the ring indicated by the arrow is much weaker, was the pattern taken at 8 days after the lumpectomy that is $4 \mathrm{~cm}$ from the follicle. Pattern 1(iii), which shows no ring, was taken 18 days after the lumpectomy and three days before the first chemotherapy. Since the lack of a ring indicates that the cancer is completely gone, there was no need for this woman to have the chemotherapy or radiation. She was already cured.

When we link these results with the early diagnosis of breast cancer using the fibre diffraction of hair or fingernails [2], we have a very accurate diagnostic test for breast cancer for women of any age. To date, $4500+$ tests have been run resulting in no false negatives. There have been some false positives, less than $1 \%$, but some of these have later been shown to be positive [3]. One such woman had put her own hair in with the test hairs she was collecting from other patients at the clinic where she worked. The diffraction results for her hair sample came up positive for 2 years in a row and were marked false positive by the doctor in charge. In the third year, her hair diffraction result was marked correct as her mammogram had shown a terminal breast cancer. She died that Christmas. If only they had accepted the fibre diffraction diagnosis 2 years earlier and not relied on mammogams.

This fibre diffraction test would eliminate the worries of women especially for those under 40 years of age. There are no problems with this test which has found breast cancers for women with family history of breast cancer in women as young as 18 . It has also eliminated the fear in others of this age group. Breast cancers in women who have a positive BRAC gene give a much sharper ring of change in the fibre diffraction test (Figure 2). This ring has been noted in women before breast cancer has been found by mammograms.

This hair diffraction test would, therefore, remove the anxiety which is driving such women to double mastectomies in their early twenties. If and when a cancer is detected, its total removal can be verified by a further hair diffraction test or tests, thus removing years of worry after surgery.

These results immediately suggested that changes in such diagnostic
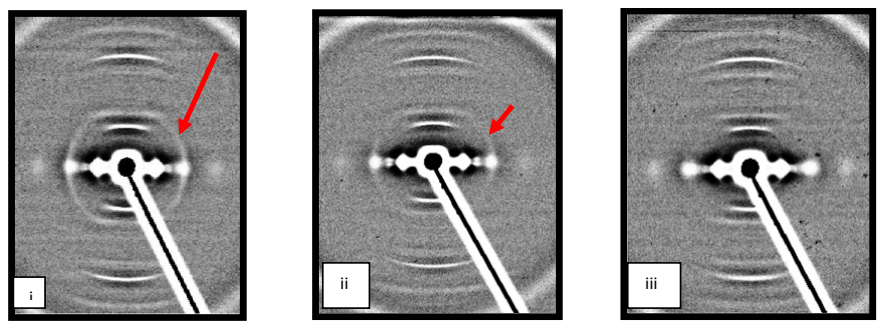

Figure 1. (i) Hair diffraction pattern 2 days before lumpectomy, (ii) Hair Hair diffraction pattern 8 days after lumpectomy, (iii) Hair Hair diffraction pattern 18 days after lumpectomy.

Correspondence to: Veronica James, Research School of Chemistry, Australian National University, Canberra ACT 0200, Australia; E-mail: veronica.james@anu.edu.au

Key words: diffraction patterns, breast cancer, prostate cancer, cure

Received: November 12, 2015; Accepted: November 27, 2015; Published: December 01, 2015 


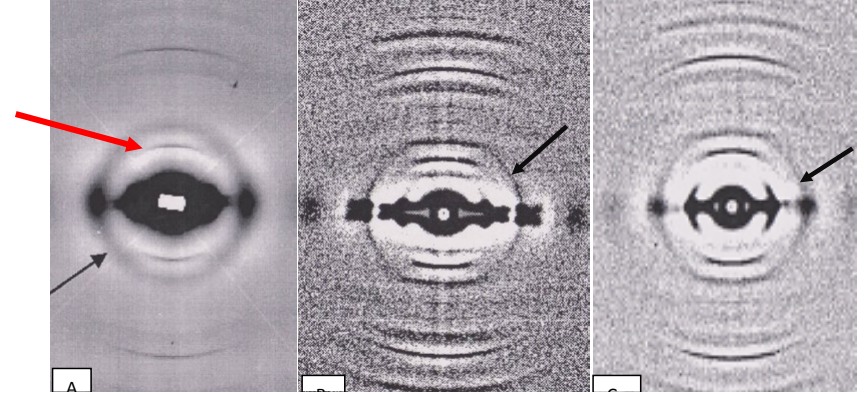

Figure 2. Figure 2A shows the normal fibre hair diffraction pattern for a woman with non-family related breast cancer. The change in the diffraction pattern is a weak diffuse ring(indicated by black arrow) superimposed on the normal diffraction pattern. The intensity of this ring is less than that of the $7^{\text {th }}$ meridional arc (indiated by the red arrow Figures $2 \mathrm{~B}$ and $2 \mathrm{C}$ are the patterns obtained for 2 Askinasi women. These rings are not diffuse but sharp. Patient $\mathrm{B}$ had the BRCA1 gene and patient $\mathrm{C}$ had the BRCA2 gene. In both cases the additional ring is sharper than that for non familial breast cancer. All these rings have the same diameters.

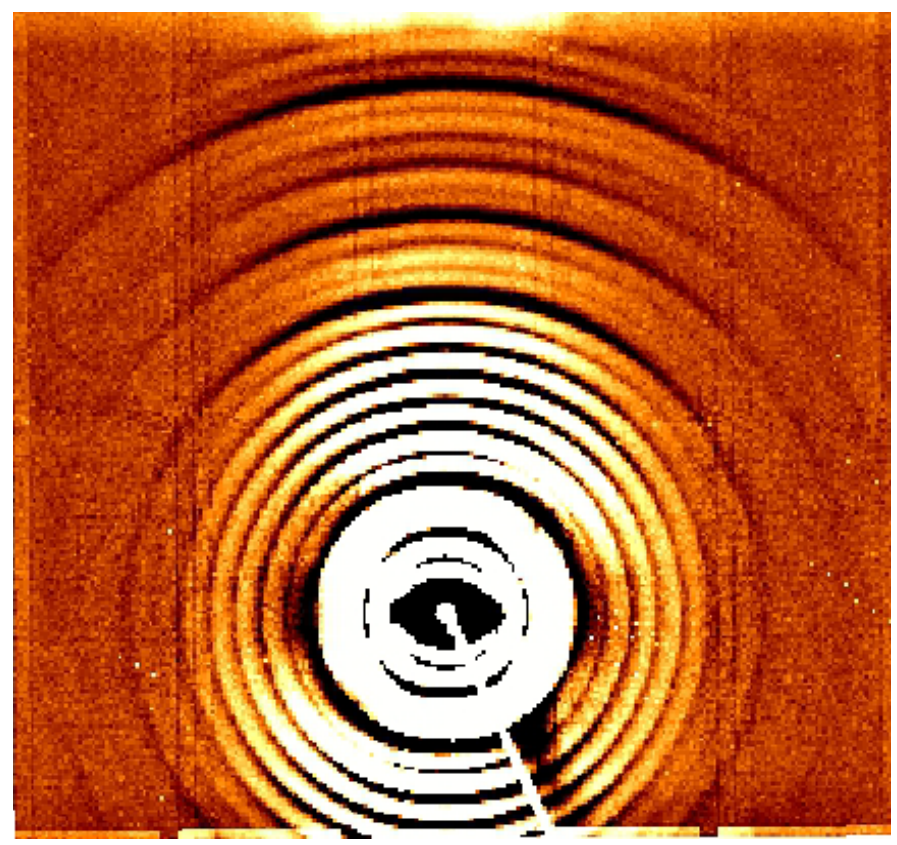

Figure 3. Normal skin pattern following cure from treatment.

tests for other cancers might also disappear if cures occur. In the course of our studies of $100+$ Australian men, we have found 7 men whose prostate cancers have been cured. All but one of these had been low grade prostate cancer patients. These six had either been treated by a prostatectomy or by chemotherapy and radiation. No rings of change associated with prostate cancer were found in their diagnostic skin biopsy fibre diffraction tests following their treatment (Figure 3).

The one remaining case had been a patient whose prostate before diagnosis was very enlarged, hard and nodular. This patient had undergone 13 separate truss biopsies of the prostate, all of which showed positive with most giving a Gleason score of 7-9, the lowest 3. A followup MRI scan and X-ray checked that the cancer was still contained in the prostate, it had not invaded. This patient, on the advice of his urologist, chose not to undergo a prostatectomy as some of the cancers were too near the surface. He chose to be treated by chemotherapy and radiation. Eight tri-monthly injections of hormone Lucrin depot 22.5 $\mathrm{mg}$ were followed by 1 month of hormone tablets, Cyproterene, $100 \mathrm{mg}$ daily. This was immediately followed by the radiotherapy consisting of 40 treatments of the highest dose attacking 7 sites in the prostate. The prostate after completion of the radiotherapy had returned to normal size and texture. No ring was found in the diffraction pattern for this patient after completion of this treatment [4].

This patient's cancer did not elevate the PSA reading, before treatment it was 3.8 and immediately after the radiotherapy finished it was 0.01 . These PSA scores indicate the uselessness of universally relying on PSA readings. The PSA reading only has meaning if the Prostate Specific Antigen is produced by the cancer and is useless otherwise. The cancers in this patient did not increase this PS antigen. The brother of this patient, who died from prostate cancer, did have a cancer that increased the PS antigen and therefore resulted in an ever increasing PSA (Figure 4).

Our last reported results indicate that most prostate cancer patients present too late [5], as the cancers of twenty out of the 30 patients in the test were high grade and their cancers had already invaded. Nineteen of these invasions were through the surface; the 20th patient's cancer had invaded through the surface and also via the lymph system.

A diffraction test involving TRAMP mice [6] has shown clear evidence that the presence of prostate cancer in such mice is clearly visible in the diffraction pattern at 3 weeks of age (Figure 5), whereas it is not able to be confirmed until 10 weeks by any other test [7].

If the fibre diffraction tests were used, from an early age, however, prostate cancers could be diagnosed when very low grade. These cancers could then be treated and the success or not of the subsequent treatments tested by follow up diffraction tests. No living in fear would be needed. This would require annual or biennial tests for Caucasian men from their late twenties. For men of negro background in the USA, it has been found that the age to start would be 18 as men of that background have been found with prostate cancer at this early age. At this stage, the age has not been set for other nationalities. Such early tests would greatly lower the death rate for prostate cancer.

Melanomas in patients also cause additional rings in skin diffraction patterns, and melanomas, if present, would be identified at the same time as prostate cancers (Figure 6). The patterns are different but unfortunately there is no way of locating the position of the melanoma from the pattern. However once again, this ring of change disappeared when the melanoma was cured but remained when it was not cured.

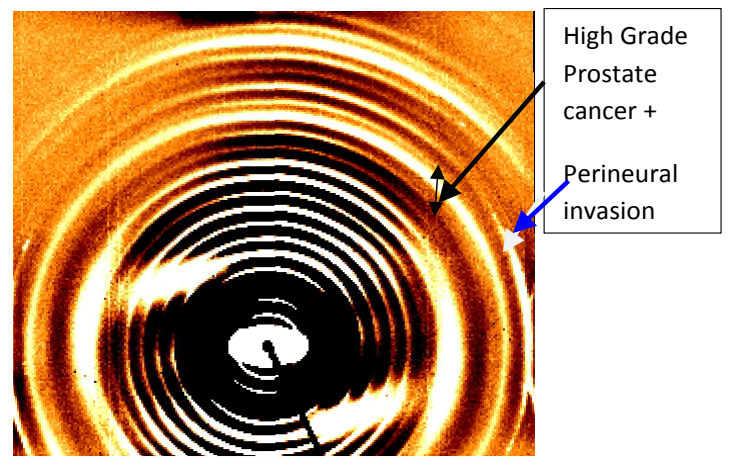

Figure 4. The ring that is associated with high grade ptostate cancer is indicated by the long black arrow, its width indicated by double end arrow. The $17^{\text {th }}$ order meridional ring indicates (blue arrow) that this cancer has invaded through the surface. A full ring through the $18^{\text {th }}$ order would indicate a lymphatic invasion 

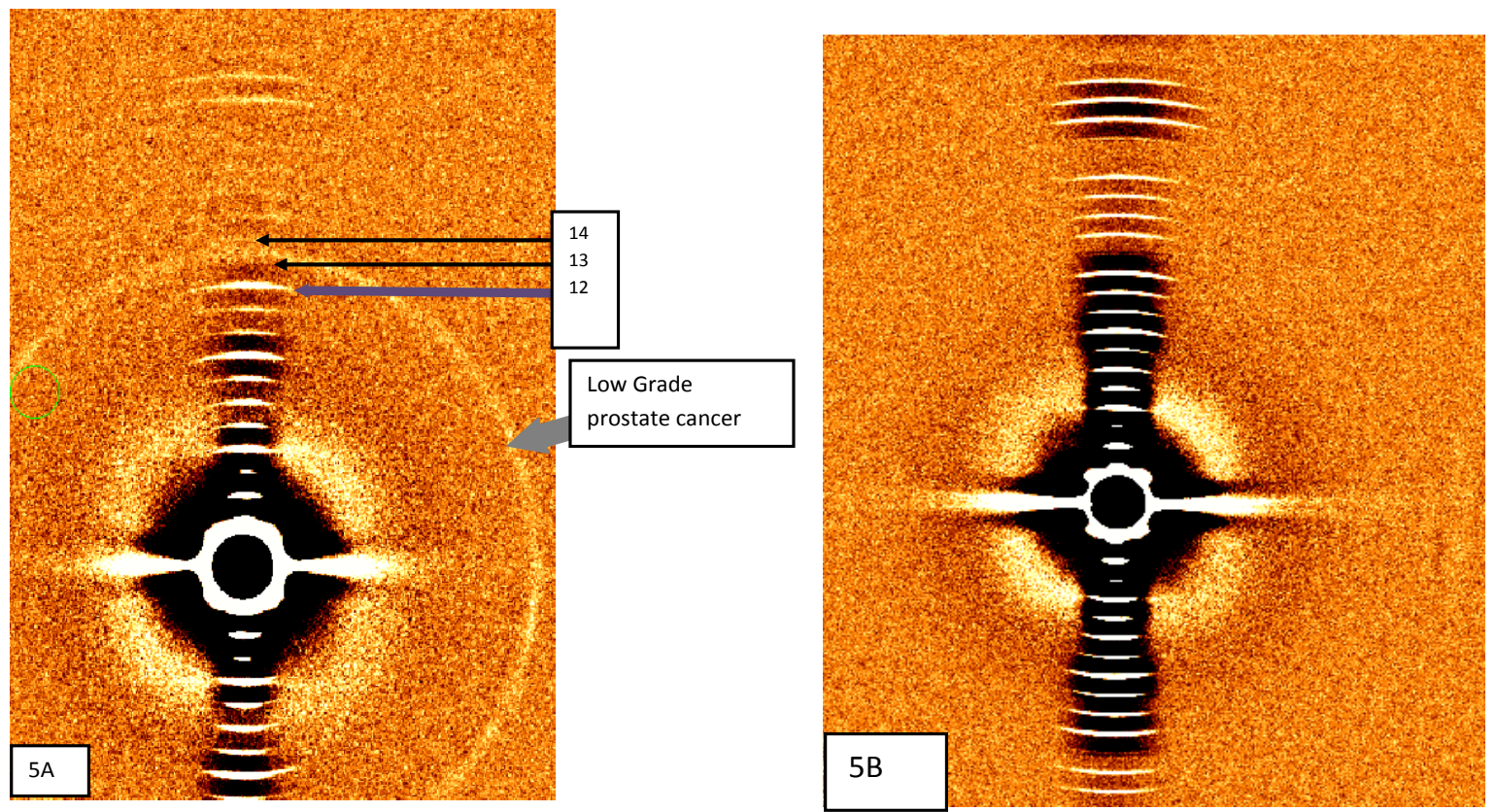

Figure 5. Figure 5A pattern taken from the tail tip of a 3 week-old Tramp mouse clearly shows the low-grade prostate cancer ring superimposed on the pattern from a normal rat-tail Figure 5B. This ring is of equal width and intensity throughout the full circle and is located between the $13^{\text {th }}$ and $14^{\text {th }}$ orders of the meridional pattern. In Figure 5B there are no extra rings in an age matched control.

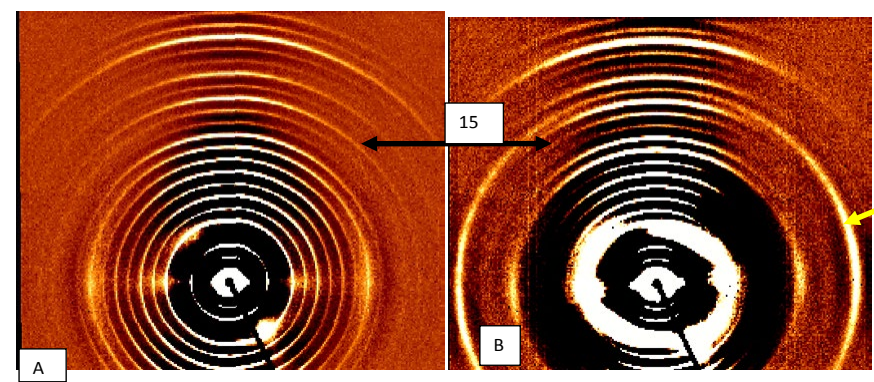

Figure 6. Figure 6A is a normal pattern for skin. Figure $6 \mathrm{~B}$ is the pattern for a person with a melanoma, The added ring for melanoma [indicated by yellow arrow] is strongest in the equatorial direction but actually passes through the $16^{\text {th }}$ meridional order.

\section{Conclusion}

As these changes in fibre diffraction tests give very early and highly accurate evidence of the presence of these cancers in the body, surely they must save lives. Early detection of any cancer is the answer to its safe removal and here we have the earliest diagnosis for cancer detection and above all proof of its successful removal. Please delay no longer!

\section{Acknowledgements}

The authors acknowledge the generous support for the use of the BioCAT Facility, Advanced Photon Source, Argonne National Laboratory and for the assistance provided by the beam-line staff of this facility. Advanced Photon Source is supported by the U.S. Department of Energy; BioCAT is supported by the National Institutes of Health.

The authors also acknowledge the generous support for the use of the Small and Wide Angle Scattering Beamline at the Australian Synchrotron, Victoria, Australia, and for the assistance provided by the beam-line and administration staff of this facility.

\section{References}

1. James V, Kearsley J, Irving T, Amemiya Y, Cookson D (1999) Using hair to screen for breast cancer. Nature 398: 33-34. [Crossref]

2. Braun-Falco O, Plewig G, Wolff HH, Burgdorf WHC (2000) Dermatology (Second, completely revisededn). Berlin, Springer: 1101. ISBN 97435405940594529.

3. James V, Corino G, Robertson T, Dutton N, Halas D, et al. (2005) Early Diagnosis of breast cancer by hair diffraction. Int J Cancer 114: 969-972. [Crossref]

4. James V (2003) False-positive results in studies of changes in fiber diffraction of hair from patients with breast cancer may not be false. J Natl Cancer Inst 95: 170-171. [Crossref]

5. James (2013) A Review of Low Angle Fibre Diffraction in the Diagnosis of Disease. $\mathrm{Br}$ J Med Med Res 3: 383-397.

6. JamesVJ (2011) Extremely Early Diagnostic Test for Prostate Cancer. J Cancer Ther 2: 377-380. [Crossref]

7. James VJ, O'Malley Ford J (2014) Fibre Diffraction Analysis of Skin Offers a Very Early and Extremely Accurate Diagnostic Test for Prostate Cancer. Journal of Cancer Research.

Copyright: (C) 2015 James VJ. This is an open-access article distributed under the terms of the Creative Commons Attribution License, which permits unrestricted use, distribution, and reproduction in any medium, provided the original author and source are credited. 